Case Report

\title{
Mitochondrial myopathy and comorbid major depressive disorder: effectiveness of dTMS on gait and mood symptoms ${ }^{2 / 2}$
}

\author{
Chiara Rapinesi, M.D. ${ }^{\text {a,b }}$, Delfina Janiri, M.D. ${ }^{\text {a }}$, Georgios D. Kotzalidis, M.D., Ph.D. ${ }^{\text {a,* }}$, Daniele Serata, M.D. ${ }^{\text {a,b }}$, \\ Antonio Del Casale, M.D. ${ }^{\text {a,c }}$, Paola Scatena, M.D. ${ }^{\text {, }}$ Claudia Dacquino, Ph.D. a , Giovanna Gentile, Ph.D. ${ }^{\text {d }}$, \\ Giovanni Manfredi, M.D. ' , Emanuela Danese, M.D. ${ }^{a}$, Ruggero Nessim Raccah, M.D. e , Roberto Brugnoli, M.D. ${ }^{\text {a }}$, \\ Gemma Callovini, M.D. ${ }^{a}$, Vittoria Rachele Ferri, M.D. ${ }^{a}$, Stefano Ferracuti, M.D. ${ }^{a}$, Abraham Zangen, Ph.D. , \\ Maurizio Simmaco, M.D. ${ }^{\text {, }}$ Gloria Angeletti, M.D. ${ }^{a}$, Paolo Girardi, M.D. ${ }^{\text {a,b }}$ \\ a NESMOS Department (Neurosciences, Mental Health, and Sensory Organs), Sapienza University of Rome School of Medicine and Psychology, Sant'Andrea Hospital, Rome, Italy \\ b Villa Rosa, Suore Hospitaliere of the Sacred Heart of Jesus, Viterbo, Italy \\ c Department of Psychiatric Rehabilitation, Fondazione Padre Alberto Mileno Onlus, Vasto, Chieti, Italy \\ d DiMA Department (Advanced Molecular Diagnosis), School of Medicine and Psychology, Sapienza University, Rome, Italy \\ e ATID Ltd Advanced Technology Innovation Distribution, Rome, Italy \\ ${ }^{\mathrm{f}}$ Department of Life Sciences, Ben Gurion University of the Negev, Be'er Sheva, Israel
}

\section{A R T I C L E I N F O}

\section{Article history}

Received 18 September 2014

Revised 26 February 2015

Accepted 5 March 2015

Available online $\mathrm{xxxx}$

\section{Keywords:}

Mitochondrial myopathy

Major depressive disorder

Comorbidity

Dorsolateral prefrontal cortex (DLPFC)

Deep transcranial magnetic stimulation (dTMS)

\begin{abstract}
A B S T R A C T
Background: Mitochondrial myopathies (MMs) often present with leukoencephalopathy and psychiatric symptoms, which do not respond to or worsen with psychiatric drugs.

Case report: A 67-year-old woman with a 10-year history of probable chronic progressive external ophthalmoplegia, an MM, had drug-resistant, anxious-depressive symptoms. Since she had never had seizures, we proposed 20 sessions of deep transcranial magnetic stimulation (dTMS) for her depression. Surprisingly, besides the expected improvement of depression, we observed marked improvement of movement disorder that lasted as long as the patient was undergoing dTMS. She also improved her performance on neuropsychological tests of executive function and cognitive speed. Depressive symptom improvement was persistent, while anxiety symptoms recurred after the end of the sessions.

Conclusions: dTMS may be an alternative antidepressant strategy in patients with MMs, provided that they are free from seizures. The mechanism of improvement of motor disturbance may relate to dorsolateral prefrontal cortex stimulation and improved executive function and needs further investigation.
\end{abstract}

(c) 2015 Elsevier Inc. All rights reserved.

\section{Introduction}

Mitochondrial deoxyribonucleic acid alterations may result in heterogeneous neuromuscular conditions, termed mitochondrial myopathies (MMs). These typically present with muscular and central nervous system symptoms, such as ptosis, weakness, muscle pain, ataxia, fainting, cramps, twitches, hyperreflexia or hyporeflexia, aberrant thermoregulation, intellectual impairment or decay, and behavioral symptoms (anxiety, depression, and/or psychosis). Onset may occur at any time, usually with neuropsychiatric dysfunction.

MMs are classified as follows: (a) mitochondrial encephalomyopathy, lactic acidosis, and stroke-like syndrome (MELAS); (b) myoclonic

\footnotetext{
Chiara Rapinesi, M.D., treated the patient, performed the stimulation and followed her up for psychiatric issues; together with Georgios D. Kotzalidis, M.D., she wrote the case report. Delfina Janiri, M.D., performed bibliographic searches, suggested genetic polymorphisms to investigate, wrote the paper with Georgios D. Kotzalidis, M.D., who organized the structure of the report and the supplement, performed bibliographic searches, suggested appropriate examinations and interviewed the patient, obtaining consent for publication of the case report. Daniele Serata, M.D., followed up the patient and wrote part of the technique; Antonio Del Casale, M.D., and Emanuela Danese, M.D., arranged for magnetic resonance imaging, interpreted the results and wrote the imaging section. Paola Scatena, M.D., made neurological diagnosis, followed up the patient for neurological aspects and wrote the part regarding histological findings. Giovanna Gentile, Ph.D., and Maurizio Simmaco, M.D., performed genetic analyses and wrote the genetics section. Claudia Dacquino, Psy.D., performed neuropsychological evaluations and wrote the neuropsychological section of the supplement and paper. Giovanni Manfredi, M.D., Roberto Brugnoli, M.D., Gemma Callovini, M.D., and Stefano Ferracuti, M.D., followed up the patient for the movement disorder and wrote the corresponding section. Ruggero Nessim Raccah, M.D., provided deep transcranial magnetic stimulation expertise and wrote part of the methods; Abraham Zangen, Ph.D., supervised the writing of the case and corrected parts of it; Gloria Angeletti, M.D., and Roberto Brugnoli evaluated the patient and collaborated with Dr. Rapinesi in her pharmacological management. Finally, Prof. Paolo Girardi, M.D., supervised the writing of the paper and coordinated the entire équipe of clinicians. All authors have seen and approved the final form of the paper.

* Corresponding author. NESMOS Department (Neurosciences, Mental Health, and Sensory Organs), Sapienza University School of Medicine and Psychology, Psychiatry Unit, Sant'Andrea Hospital, Rome, Italy. Tel.: + 39-0633775951; fax: + 39-0633775342.

E-mail address: giorgio.kotzalidis@uniroma1.it (G.D. Kotzalidis).
} 
epilepsy and ragged-red fibers (MERRF); (c) Kearns-Sayre syndrome (KSS); and (d) chronic progressive external ophthalmoplegia (CPEO). Their complex genetics and mechanisms are still unknown and their diagnosis is often problematic due to symptom overlap between the various MMs, and this is in part reflected by their genetic heterogeneity (see Supplement).

Psychiatric comorbidity is $70 \%$, most commonly involving mood and anxiety disorders, which may precede the clinical onset of MM [1]. Psychiatric-mitochondrial dysfunctions are bidirectionally linked [2].

MM treatments are few and comorbid psychiatric disorders often resist to drugs; psychiatric drugs, like antipsychotics and valproate, which inhibit the respiratory chain, may even worsen them [3].

Deep transcranial magnetic stimulation (dTMS) recently received Food and Drug Administration approval for adults with depression responding poorly to adequate treatment. We administered add-on dTMS to a patient with CPEO-like MM and severe depressive/anxiety symptoms, which promptly responded, while unexpectedly, gait disturbance improved transiently.

\section{Case description}

In January 2013, we saw a 67-year-old woman with hearing loss, standing/walking inability, fatigue, free-floating anxiety, crying, apathy, avolition, obnubilation, depression, and active social withdrawal. She scored 24 on the Hamilton Depression Rating Scale (HDRS) and 25 on the Hamilton Anxiety Rating Scale (HARS).

Physical examination revealed reduced gait synkinesias, right plastic hypertonus, bilateral palpebral ptosis, and inferior facial deficit, as well as nystagmus, blunted medial plantar and ankle jerk reflexes, left motor dysmetria, tremor, difficulty swallowing, and dysarthria. She is $162 \mathrm{~cm}$ tall and weighs $64 \mathrm{~kg}$.

\subsection{Past medical history}

Between 2003 and 2004, the patient developed increasingly severe muscular symptoms, bilateral palpebral ptosis, visual disturbance, dysphagia, and dysphonia, but brain and spine magnetic resonance imaging (MRI) and electroencephalogram (EEG) showed no abnormalities. In 2005, she was diagnosed with unspecified MM after muscular biopsy. Venous lactic acid was normal, but rose to $11.7 \mathrm{mEq} / \mathrm{L}$ under effort. Electromyography (EMG) showed myopathy with intact nervous transmission. Biopsy and EMG details are provided in Supplement. Motor and sensitive neurography showed no abnormalities.

Gait instability/impaired deambulation started out in 2008 , needing rehospitalization. Repeat EEG showed bilateral temporal theta waves, echocardiogram mitral and tricuspid prolapse, and electrocardiogram repolarization abnormalities with diastolic conduction deficit. A single photon emission computerized tomography showed no abnormality. Repeat MRI showed chronic leukoencephalopathy with hyperintensities in the right frontal corona radiata, while repeat EMG confirmed preceding results. Audiometry showed high-frequency sensorineural hearing loss.

Her family history was negative for psychiatric disorders, but her mother, who had died at the age of 80 years, had developed severe ptosis, diabetes, and hypoacusis since the age of 71 years, while one of her two daughters currently reports fatigue. No personal or family history for epilepsy.

\subsection{Past psychiatric history}

Patient developed anxiety and depression in 1994 and underwent five hospitalizations. Her psychiatric symptoms progressively worsened, and in 2001, she had her first panic attacks. Past adequately dosed and sufficiently prolonged drug treatment (at least 2 months for each drug) included paroxetine, alprazolam, haloperidol, sulpiride, risperidone, venlafaxine, escitalopram, mirtazapine, valproate, zolpidem, and desmethyldiazepam, which helped little. She suffered 10 nonpsychotic depressive episodes lasting 20 weeks each; some of them were characterized by suicidal ideation, but she never attempted suicide.

Due to the progressive nature of MMs, we decided to carry out another MRI to assess the progression of leukoencephalopathy and to subject her to neuropsychological cognitive testing because MMs are often associated with mental decline. Furthermore, in an attempt to pinpoint her diagnosis, we performed genetic analysis of the most frequent genetic culprits of MMs (details in Supplement).

Since psychiatric symptoms were unresponsive, we proposed her add-on dTMS because dorsolateral prefrontal cortex (DLPFC) stimulation showed positive effects in depression. At that time, the patient was on sulpiride, $75 \mathrm{mg} /$ day, taken continuously during the last 2 years; on risperidone, $2 \mathrm{mg} /$ day since 18 months; and on venlafaxine, $150 \mathrm{mg} /$ day in the last 22 months.

\subsection{Results of examinations and treatments}

\subsubsection{MRI}

Repeat MRI showed scattered, bilateral, circumscribed white matter hyperintensities, presumably due to reactive gliosis, along with temporoinsular and parietal subarachnoid space dilation (Supplementary Figure).

\subsubsection{Neuropsychological assessment}

Neuropsychological assessment was conducted at baseline and after 4 weeks of dTMS (detailed in Supplement).

Patient's baseline general intelligence level was normal. She showed no deficit in verbal memory, language, and motor planning but showed impaired long-term visuospatial memory, slow cognitive processing speed, and slight impairment on the Wisconsin Card Sorting Test. After 4 weeks of dTMS, her performance on neuropsychological tests was without any deficit, with improvements on all investigated areas.

\subsubsection{Genetic analyses}

Patient was negative for the most common MELAS and MERRF polymorphisms (details in Supplement). Due to limited compatibility with other MMs, we diagnosed an atypical CPEO, based on considerations reported in Supplement. Briefly, she did not fit in the MELAS or MERRF categories, but she shared KSS and CPEO features, being incompatible with a KSS diagnosis due to her late onset and lack of pigmentary retinopathy. We attributed psychological symptoms to her underlying medical condition.

\subsection{4. dTMS treatment}

For dTMS, we used Brainsway's H1 coil deep TMS System (see Supplement). The cycle consisted of five consecutive session days per week for four consecutive weeks, totaling 20 sessions. dTMS was well tolerated.

Depression, anxiety (HARS, 16; HDRS, 11), and deambulation improved after two sessions, continuing through the remaining treatment period. Remarkably, the patient was able to walk at the second session. Anxiety resisted to improvement more than depression; HDRS reached 7 at day 7 and thus remained through the remaining sessions, while HARS ranged 12-13 during treatment weeks 1-4 but returned to clinically significant levels (17) 1 week after the end of dTMS.

Gait improvement preceded the one in depression and was apparent as long as the patient received dTMS; it was lost after the end of the sessions (see Supplement).

The patient signed free, informed consent for all treatments and for case publication.

\section{Discussion}

Although comorbid psychiatric illness is increasingly recognized in patients with mitochondrial disorders, little attention focuses on psychiatric symptomatology as the primary clinical presentation. MMs 
may present with mood disorder, cognitive impairment, psychosis, and anxiety [4,5]. Psychiatric comorbidity may impair patients' quality of life and requires treatment [5]. However, psychiatric drugs may further worsen the underlying MM, as they interfere with mitochondrial function $[3,6,7]$. Hence, there is a need to identify treatments free from untoward effects.

While our patient had never responded to psychopharmacological treatment, she responded promptly to dTMS. As expected, her depression responded most prominently, but surprisingly, her gait disturbance improved even earlier. However, while the antidepressant effect persisted, effects on anxiety and deambulation were lost after the last session.

It is suggested that DLPFC activation may be related with improvement of depression [8], but no data exist for DLPFC and movement quality. We might suppose that the observed improvement in motor performance was secondary to dTMS-induced DLPFC stimulation.

dTMS is noninvasive and with few side effects; however, it is contraindicated in epilepsy, which is ubiquitous in MERRF and frequent in MELAS [9]. Genetics and history excluded epilepsy and allowed dTMS trial.

The currently unclear relationships between psychiatric disorders and mitochondrial dysfunction are multiple and bidirectional $[2,10]$ and are neglected in clinical practice. Psychiatrists could consider MM when faced with treatment resistance and assess mitochondrial function. Other clinicians could view psychiatric symptoms emerging during MMs not as self-standing entities but rather as embedded in the whole clinical picture.

\section{Financial and Competing Interests Disclosure}

In the past 2 years, Paolo Girardi has received research support from Lilly, Janssen, and Springer Healthcare; has participated in Advisory Boards for Lilly, Otsuka, Pfizer, Schering, and Springer Healthcare; and received honoraria from Lilly and Springer Healthcare. Ruggero N. Raccah is scientific consultant to ATID Ltd, distributor of deep r-TMS (Brainsway) technology in Italy. All other authors of this paper have no relevant affiliations or financial involvement with any organization or entity with a financial interest in or financial conflict with the subject matter or materials discussed in the manuscript. This includes employ- ment, consultancies, honoraria, stock ownership or options, expert testimony, grants or patents received or pending, or royalties.

This work has not been supported by any funding.

\section{Acknowledgments}

The authors wish to thank Ms. Mimma Ariano, Ms. Ales Casciaro, Ms. Teresa Prioreschi, and Ms. Susanna Rospo, Librarians of the Sant'Andrea Hospital, School of Medicine and Psychology, Sapienza University, Rome, for rendering precious bibliographical material accessible, as well as their Secretary Lucilla Martinelli for her assistance during the writing of the manuscript.

\section{Appendix A. Supplementary data}

Supplementary data to this article can be found online at http://dx. doi.org/10.1016/j.genhosppsych.2015.03.002.

\section{References}

[1] Fattal O, Link J, Quinn K, Cohen BH, Franco K. Psychiatric comorbidity in 36 adults with mitochondrial cytopathies. CNS Spectr 2007;12:429-38.

[2] Manji H, Kato T, Di Prospero NA, Ness S, Beal MF, Krams M, et al. Impaired mitochondrial function in psychiatric disorders. Nat Rev Neurosci 2012;13:293-307.

[3] Anglin R, Rosebush P, Mazurek M. Psychotropic medications and mitochondrial toxicity. Nat Rev Neurosci 2012;13:650.

[4] Lacey CJ, Salzberg MR. Obsessive-compulsive disorder with mitochondrial disease. Psychosomatics 2008;49:540-2.

[5] Anglin RE, Garside SL, Tarnopolsky MA, Mazurek MF, Rosebush PI. The psychiatric manifestations of mitochondrial disorders: a case and review of the literature. J Clin Psychiatry 2012;73:506-12.

[6] Anglin RE, Rosebush PI, Mazurek MF. Treating psychiatric illness in patients with mitochondrial disorders. Psychosomatics 2010;51:179.

[7] Hroudová J, Fišar Z. In vitro inhibition of mitochondrial respiratory rate by antidepressants. Toxicol Lett 2012;213:345-52.

[8] Levkovitz Y, Harel EV, Roth Y, Braw Y, Most D, Katz LN, et al. Deep transcranial magnetic stimulation over the prefrontal cortex: evaluation of antidepressant and cognitive effects in depressive patients. Brain Stimul 2009;2:188-200.

[9] Valk J, van der Knaap MS. Magnetic resonance of myelin, myelination, and myelin disorders. Chapter 24: Mitochondrial Leukoencephalopathy. Berlin: Springer Verlag; 1989 130-6.

[10] de Sousa RT, Uno M, Zanetti MV, Shinjo SM, Busatto GF, Gattaz WF, et al. Leukocyte mitochondrial DNA copy number in bipolar disorder. Prog Neuropsychopharmacol Biol Psychiatry 2014;48:32-5. 